\title{
Parallel Interference Cancellation with MMSE Equalization for the Downlink MC-CDMA Systems
}

\author{
B.A. Al-fuhaidi ${ }^{*}$, H.E.A. Hassan ${ }^{\dagger}$, M.M. Salah ${ }^{\ddagger}$, S.S. Alagooz ${ }^{\S}$
}

\begin{abstract}
The Multi-Carrier Code Division Multiple Access (MC-CDMA) is becoming a very attractive multiple access technique for high-rate data transmission in the future wireless communication systems. MC-CDMA systems transmitting over multipath channels suffer from intersymbol interference (ISI) and multiple access interference (MAI). Recently, MCCDMA with equalization has attracted much attention for its ability to obtain an excellent performance even in strong frequency selective fading channels. In this paper, the proposed architecture uses the minimum mean square error (MMSE) with parallel interference cancellation (PIC) for downlink MC-CDMA system and compared with another system based on the rake receiver with PIC for downlink MC-CDMA system. A comparison between such architectures is presented. The effect of the tentative decision functions, the number of cancelled users and the effect of the loading users on the performance of the two proposed receivers are discussed and presented in this paper. Simulation results show that the combination of tentative decisions with PIC and equalization provides an efficient solution to suppress the MAI and ISI in downlink MC-CDMA systems over frequency selective fading channels.
\end{abstract}

Keywords: Downlink MC-CDMA, Decision functions, Equalization, RAKE-PIC, MMSE

\section{Notations}

The symbols $(.)^{H},(.)^{T}$, and $(.)^{-1}$ designate complex conjugate transposition, transposition of a matrix, and the inverse of a matrix, respectively. Vectors and matrices are represented in boldface. $\boldsymbol{F}^{\mathbf{- 1}}$ and $\boldsymbol{F}$ denote the inverse fast Fourier transform and the fast Fourier transform operators, respectively.

\section{Introduction}

Multicarrier code-division multiple-access (MC-CDMA) system has been proposed as one of the candidates for the future generations of wireless communications because of its attractive features such as high data rate, high capacity, and low complexity of implementation due to using fast Fourier transform (FFT)-based carrier modulation[1,2,6]. In these schemes each user is assigned to a unique identification code sequence and the transmitted signal is split in different subcarriers. etc.[1,3,4,8]. MC modulation and demodulation can be easily

\footnotetext{
*Yemeni Armed Forces, belalarh@gmail.com

† Egyptian Armed Forces, hossameldin_aboubakr@hotmail.com

Egyptian Armed Forces, 
implemented using inverse fast Fourier transform (IFFT) and fast Fourier transform (FFT) algorithms, respectively, depending on the spreading, frequency mapping and detection strategy [3].

MC-CDMA communication system is inherently interference limited. As all users spread their transmissions over a common bandwidth, a dominant impairment is the interference between users, referred to as the MAI. In addition, in a frequency selective fading channel the bit error rate (BER) performance of a single carrier transmission significantly degrades due to severe ISI. Direct sequence code division multiple access (DS-CDMA) can exploit the channel frequency selectivity by the use of coherent rake receiver that resolves the propagation paths having different time delays and coherently combines them to achieve the path diversity effect [5,7]. Recent studies have been shifted from DS-CDMA to multi-carrier (MC) transmission techniques to overcome severe frequency selectivity of the fading channel and suppress the interference effect in a wireless channel [6,9].

Moreover, after equalization, inter-chip interference (ICI) arises, and dispreading may not properly restore the original signal [5]. Suppressing the MAI, the ICI and the ISI will reduce thetransmitted power required to meet the target signal-to-noise ratio (SNR), and enable more channels to be transmitted with a limited power.

In order to combat the MAI, the ICI, and the ISI, various receiver architectures have been proposed. The RAKE receiver is commonly used for the detection of transmitted symbols in MC-CDMA systems. However, in practice, the number of fingers in the RAKE receiver is limited due to the hardware complexity [7]. Such limited number of RAKE receiver fingers degrades the receiver performance when the multipath effect is severe. Recently, single carrier block transmission with frequency domain minimum mean square error (MMSE) equalization has proved to be a promising candidate for broadband wireless communications, especially when the implementation issues such as power consumption and system complexity are taken into consideration $[7,10]$.

Equalization with MC-CDMA can be used to compensate for ISI resulting from timedispersive channels. The price to be paid is a reduction in the data rate due to the insertion of the cyclic prefix for MC-CDMA systems [2,6]. However, the presence of some residual ICI after frequency domain equalization (FDE) degrades the orthogonality among the spreading codes, and hence the performance deteriorates [11-13]. For the reduction of the ICI, a joint frequency domain equalization and frequency domain ICI cancellation (FD-ICI) has been studied [11-13]. In [11-13], the ICI is generated and subtracted after the FDE in the frequency domain. With regard to nonlinear solutions, block decision feedback equalizers for cyclic prefix CDMA (CP-CDMA) systems and large multiple input multiple output (MIMO) systems have been proposed $[14,15]$.

The objective of this paper is to enhance the performance of the downlink MC-CDMA systems by mitigating the MAI and the ISI. A hybrid algorithm of detection comprising both linear equalization and tentative decisions with PIC is suggested and studied in the paper. Two efficient interference cancellation architectures RAKE-PIC and MMSE-PIC are proposed to be used in this hybrid algorithm. In addition, the mathematical form of the MAI before equalization (or before the RAKE receiver) is simpler than its mathematical form after equalization [16,17]. Thus, the PIC scheme composed of the regeneration and subtraction of the MAI before equalization has a lower complexity and is more appropriate in implementation [17]. The efficiency of all algorithms comes from the frequency domain implementation of all filters. The effective of the tentative decision (hard, tanh, null zone, unit clipper and soft) functions on the two proposed receivers are studied in this paper and show the advantage of any decision function to each other. All of the proposed algorithms are studied and compared to existing algorithms.

The remainder of this paper is organized as follows. Section 2 investigates some different decision functions. In Section 3, the system model of downlink MC-CDMA is presented. In 
Section 4, the proposed two interference cancellation architectures are presented. The computer simulations and the conclusions are given in Sections 5 and 6, respectively.

\section{Decision Functions}

The performance of PIC depends on the tentative decision function used. So, due to error propagation, PIC with a hard decision function may perform worse than PIC with linear or soft decision functions [18]. Hard-decision interference cancellation can completely cancel interference only when the hard decisions made are correct which is not the case in all decisions.

The most popular decision functions are:

\subsection{The hard decision function}

$y=f_{d e c}(x)=\left\{\begin{aligned} 1, & x \geq 0 \\ -1, & x<0\end{aligned}\right.$

It makes a hard decision in the favor of one of the two possible symbols.

\subsection{The null zone decision function}

$y=f_{d e c}(x)=\left\{\begin{array}{rr}1, & x>C_{n}, \\ 0, & x \in\left[-C_{n}, C_{n}\right], \\ -1, & x<-C_{n} .\end{array}\right.$

It makes a hard decision when the soft bit estimate lies outside the interval $\left[-C_{n}, C_{n}\right]$, and sets the decision result to zero when the soft bit estimate lies inside this interval. $\mathrm{C}_{\mathrm{n}}$ is the null zone decision threshold ( $0 \leq C_{n} \leq 1$ ) $[18,19]$.

\subsection{The linear decision function}

$y=f_{d e c}(x)=x$.

It performs worse than the other decision functions.

\subsection{The unit clipper decision function}

$y=f_{d e c}(x)=\left\{\begin{array}{rr}\mathbf{1}, & x>1 \\ x & x \in[-\mathbf{1}, \mathbf{1}] \\ -\mathbf{1}, & x<-1\end{array}\right.$

It makes a soft bit decision when the soft bit estimate lies inside the interval $[-1,1]$ to avoid the propagation of errors, and makes a hard decision when the soft bit estimate lies outside the interval $[-1,1]$ to avoid the noise enhancement [20].

\subsection{The tanh decision function}

$\mathbf{y}=\mathbf{f}_{\operatorname{dec}}(\mathbf{x})=\tanh (x)=\frac{\sinh (x)}{\cosh (x)}$

where $\tanh$ is hyperbolic tangent function. 
It is adopted as the optimum decision function for nonfrequency selective fading channels with the MAI modeled as AWGN in the systems having a large number of users.

\section{System Model}

The downlink MC-DMA block transmission with $\mathrm{K}$ active users over a frequency selective fading channel is considered. A schematic diagram of the baseband block transmission system is depicted in Fig. 1. Each user transmits BPSK information symbols. Those symbols are spread using a certain spreading code. After spreading, the resulting signal is scrambled using a complex scrambling sequence, after that the inverse fast Fourier transform(IFFT) applied to the resulting signal and a cyclic prefix of $\mathrm{N}_{\mathrm{CP}}$ chips is added at the beginning of each block. The length of the cyclic prefix must be greater than the maximum excess delay of the channel to accommodate for the interblock interference (IBI). At the receiver, the cyclic prefix is removed and the received symbols are sent to an FFT block to demultiplex the multicarrier signal.

The baseband channel response can, then, be expressed as follows [18]:

$h(t)=\sum_{l=0}^{L-1} h_{1} \delta\left(t-\tau_{1}\right)$,

where $h_{1}$ and $\tau_{1}$ represent the complex fading and the propagation delay of the lth path, and $\mathrm{L}$ is the number of multipath components of the channel impulse response $h$. In this paper, we assume block fading, where the path gains stay constant over each block duration.

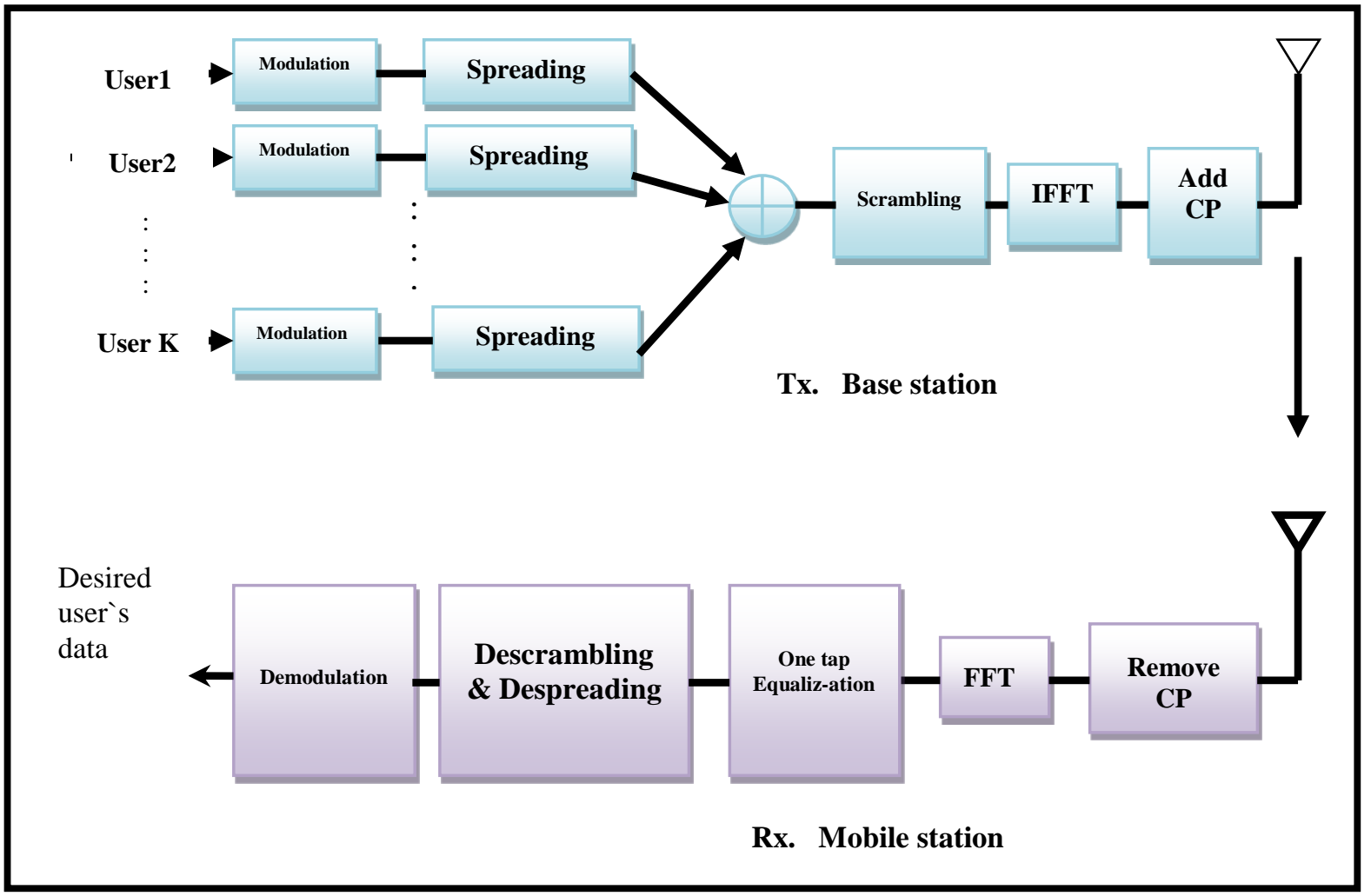

Fig. 1 Downlink MC-CDMA System

The received block after removal of cyclic prefix can be formulated as:

$\mathbf{r}=\mathbf{H}_{\mathrm{C}} \mathbf{d}+\mathbf{n}$, 
where $\mathbf{d}$ is an $\mathrm{NM} \times 1$ vector representing the block of the transmitted chip sequence, $\mathbf{r}$ is the received vector, $\mathbf{n}$ is the additive noise, and $\mathbf{H}_{\mathrm{C}}$ is an $\mathrm{NM} \times \mathrm{NM}$ circulant matrix describing the multipath channel. $\mathbf{H}_{\mathrm{C}}$ can be written as follows:

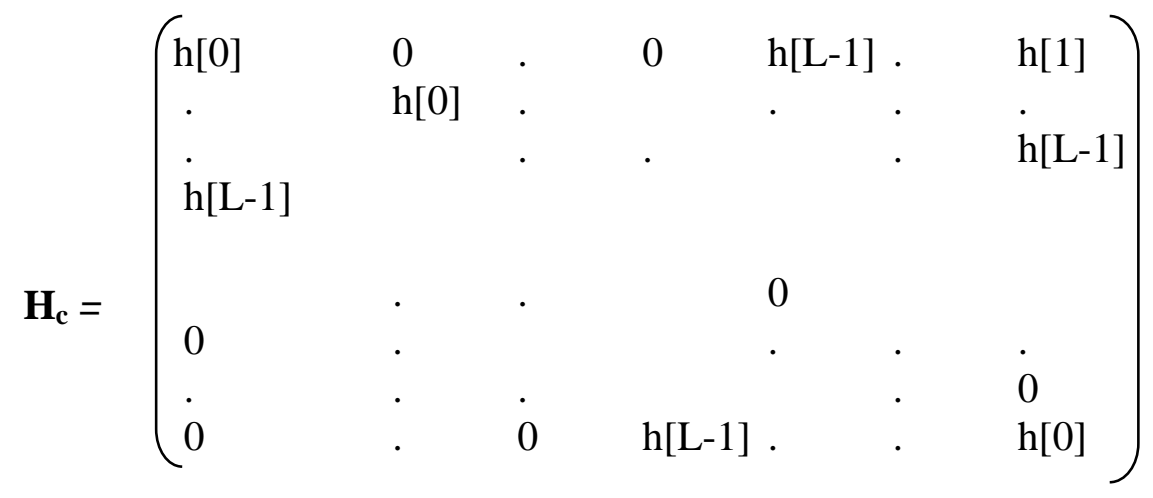

The vector $\mathbf{d}$ can be represented as:

$$
\mathbf{d}=\boldsymbol{F}^{-1} \mathbf{C S b}
$$

where $\boldsymbol{F}^{\mathbf{- 1}}$ is an NM $\times$ NM Inverse Fast Fourier transform, $\mathbf{C}$ is an $\mathrm{NM} \times \mathrm{NM}$ scrambling code matrix, $\mathbf{S}$ is an $\mathrm{NM} \times \mathrm{KM}$ block diagonal matrix whose diagonals contain the spreading vectors, and $\mathbf{b}$ is a $\mathrm{KM} \times 1$ vector consisting of the users' data. The structures of the individual components in Eq. (9) are given bellow [21]:

$$
\begin{aligned}
& S=\operatorname{diag}[\bar{S}, \bar{S}, \ldots, \bar{S}], \\
& \bar{S}=\left[s_{1}, s_{2}, \ldots, s_{K}\right], \\
& s_{K}=\left[s_{K}(0), s_{K}(1), \ldots, s_{K}(N-1)\right]^{T}, \\
& \mathbf{b}=\left[\mathbf{b}^{\mathrm{T}}(\mathbf{1}), \mathbf{b}^{\mathrm{T}}(2), \ldots, \mathbf{b}^{\mathrm{T}}(\mathrm{M})\right], \\
& \mathrm{C}=\operatorname{diag}[\mathbf{c}(\mathbf{1}), \mathbf{c}(2), \ldots, \mathbf{c}(\mathrm{NM})], \\
& \mathbf{b}(\mathbf{m})=\left[\mathrm{A}_{1} \mathbf{b}_{1}(\mathbf{m}), \mathrm{A}_{2} \mathbf{b}_{2}(\mathbf{m}), \ldots, \mathrm{A}_{\mathrm{K}} \mathbf{b}_{\mathrm{K}}(\mathbf{m})\right]^{\mathrm{T}},
\end{aligned}
$$

where $s_{K}$ is the spreading code of the kth user. Equation (7) can be written in terms of the MAI as follows:

$$
\mathbf{r}=\mathbf{H}_{\mathbf{C}} \boldsymbol{F}^{-1} \mathbf{C S}_{\mathbf{d e s}} \mathbf{b}_{\text {des }}+\mathbf{H}_{\mathbf{C}} \boldsymbol{F}^{-1} \mathbf{C U b}_{\text {int }}+\mathbf{n}=\underbrace{\mathbf{H}_{\mathbf{C}} \mathbf{d}_{\text {des }}}_{\text {Useful diversity }}+\underbrace{\mathbf{H}_{\mathbf{C}} \mathbf{d}_{\text {int }}}_{\text {MAI }}+\underbrace{\mathbf{n}}_{\text {Noise }},
$$

where $\mathbf{b}_{\text {des }}$ is an $M \times 1$ vector consisting of the desired user's bits, $\mathbf{b}_{\text {int }}$ is a $(K-1) M \times 1$ vector consisting of the interfering users' bits, $\mathbf{S}_{\mathrm{des}}$ is an $N M \times M$ matrix consisting of the spreading code of the desired user, and $\mathbf{U}$ is an $N M \times(K-1) M$ matrix consisting of the spreading codes of the interfering users.

From Eq. (16), it is found that only the first term contains the desired data, the second term is due to the MAI, and the third term is a noise term. 


\section{Interference cancellation for Downlink MC-CDMA}

\subsection{RAKE Receiver with PIC}

In this section, the first suggested scheme of the interference cancellation is introduced. This scheme uses the RAKE receiver and PIC to estimate, regenerate, and cancel all the interfering users. Then, the RAKE receiver is used to provide the enhanced desired user's data. This scheme is called (RAKE-PIC). It is shown in Fig. 2. The steps of the RAKE-PIC algorithm can be summarized as follows:

- The cyclic prefix is removed from the received signal.

- After removing the cyclic prefix, the FFT is applied to the received signal.

With the aid of Eq. (16), we get:

$$
R_{T}=E D_{\text {des }}+E D_{\text {int. }}+\mathbf{N}
$$

where $\mathbf{E}$ is a diagonal matrix containing the FFT of the circulant sequence of $\mathbf{H}_{\mathbf{c}}$ and $\mathbf{D}_{\mathbf{d e s}}, \mathbf{D}_{\text {int }}$ and $\mathbf{N}$ are the Fourier transforms of $\mathbf{d}_{\mathbf{d e s}}, \mathbf{d}_{\text {int }}$, and $\mathbf{n}$, respectively.

- The resulting signal after the FFT is first sent to the frequency domain channel estimator which estimates the channel coefficients.

- The estimate of the channel coefficients is used at stage one to estimate the symbols of the interfering users with FD-RAKE receiver. The estimates of the symbols obtained here are the first decision made, and we refer to this as a tentative decision. This step can be written as follows:

$$
\hat{\mathbf{b}}_{\text {int }}=\boldsymbol{f}_{\text {dec }}\{\mathbf{U}^{\mathrm{T}} \mathbf{C}^{\mathbf{H}} \underbrace{E^{\mathrm{H}} \mathbf{R}_{\mathrm{T}}}_{\text {Rake Receiver }}\},
$$

where $f_{\text {dec }}($.$) is the tentative decision function (tanh decision is considered).$

- The estimates of the interfering users' symbols are used with the channel estimate to regenerate the interfering users' signals (MAI) as follows:

$$
\mathbf{R}_{\text {MAI }}=E F^{-1}\left(C U \hat{b}_{\text {int }}\right) \text {, }
$$

- The MAI is then subtracted from $\mathbf{R}$ to get the frequency domain interference free signal as follows:

$$
\mathbf{Z}=\mathbf{R}-\mathbf{R}_{\mathbf{M A I}},
$$

- A better estimate of the symbols of interest can be obtained by applying the RAKE detection on the interference free signal $\mathbf{Z}$ as follows:

$$
\hat{\mathbf{b}}_{\text {des }}=\boldsymbol{f}_{\text {dec }}\left\{\mathbf{S}_{\mathrm{des}}^{\mathrm{T}} \mathbf{C}^{\mathrm{H}} \boldsymbol{E}^{\mathrm{H}} \mathbf{Z}\right\}
$$

where $f_{\text {dec }}($.$) is a hard decision function.$

The main advantage of this receiver lies in its low complexity as compared to the other suggested schemes. However, the performance of this receiver deteriorates as the number of users increases. This can be explained by the fact that, for heavy loads, the RAKE receiver sees too much interference which makes its decisions about interfering users unreliable. 


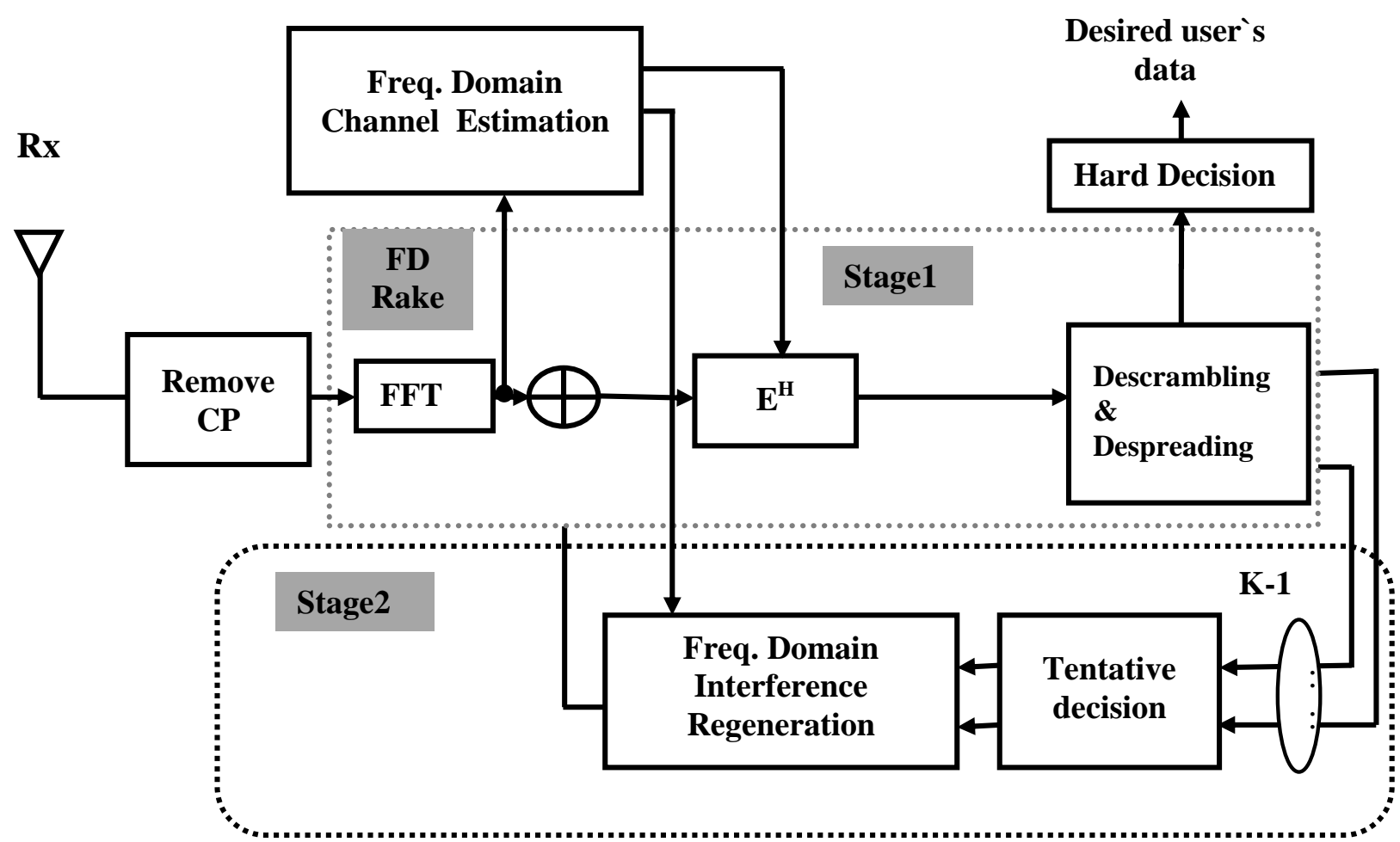

Fig. 2 Structure of the RAKE-PIC for downlink MC-CDMA systems.

\subsection{MMSE Equalizer with PIC}

In this section, we suggest the combination of both MMSE equalization and PIC to form a new interference cancellation scheme which mitigates the interference in downlink MCCDMA systems as shown in Fig. 3.

The proposed scheme consists of two parts. In the first part, MMSE equalizer is used to mitigate the effect of frequency-selective channel and give the initial data estimates of all users. In the second part, PIC is used to regenerate, and cancel MAI in frequency domain before equalization. Finally, the MMSE equalizer is used to provide the desired user's data. The suggested scheme is called MMSE-PIC. Its algorithm can be summarized as follows:

- The cyclic prefix is removed from the received signal.

- The FFT is applied on the received signal as in Eq. (17).

- The resulting signal after FFT is first sent to the frequency domain channel estimator which estimates the channel coefficients.

- The MMSE equalizer is used to estimate the sequence of the interfering users (MAI). This step can be written as:

$$
\hat{\mathbf{b}}_{\text {int }}=\boldsymbol{f}_{\text {dec }}\left\{\boldsymbol{U}^{T} \boldsymbol{C}^{H} \mathbf{W}_{\mathrm{MMSE}} \mathbf{R}_{\mathrm{T}}\right\} \text {, }
$$

The MMSE equalizer operator is given by:

$$
W_{M M S E}=\left(E^{H} E+(1 / S N R) I\right)^{-1} E^{H}
$$

- The estimates of the interfering users' symbols coupled with channel estimation are used to regenerate the interfering users' signal (MAI) as follows: 


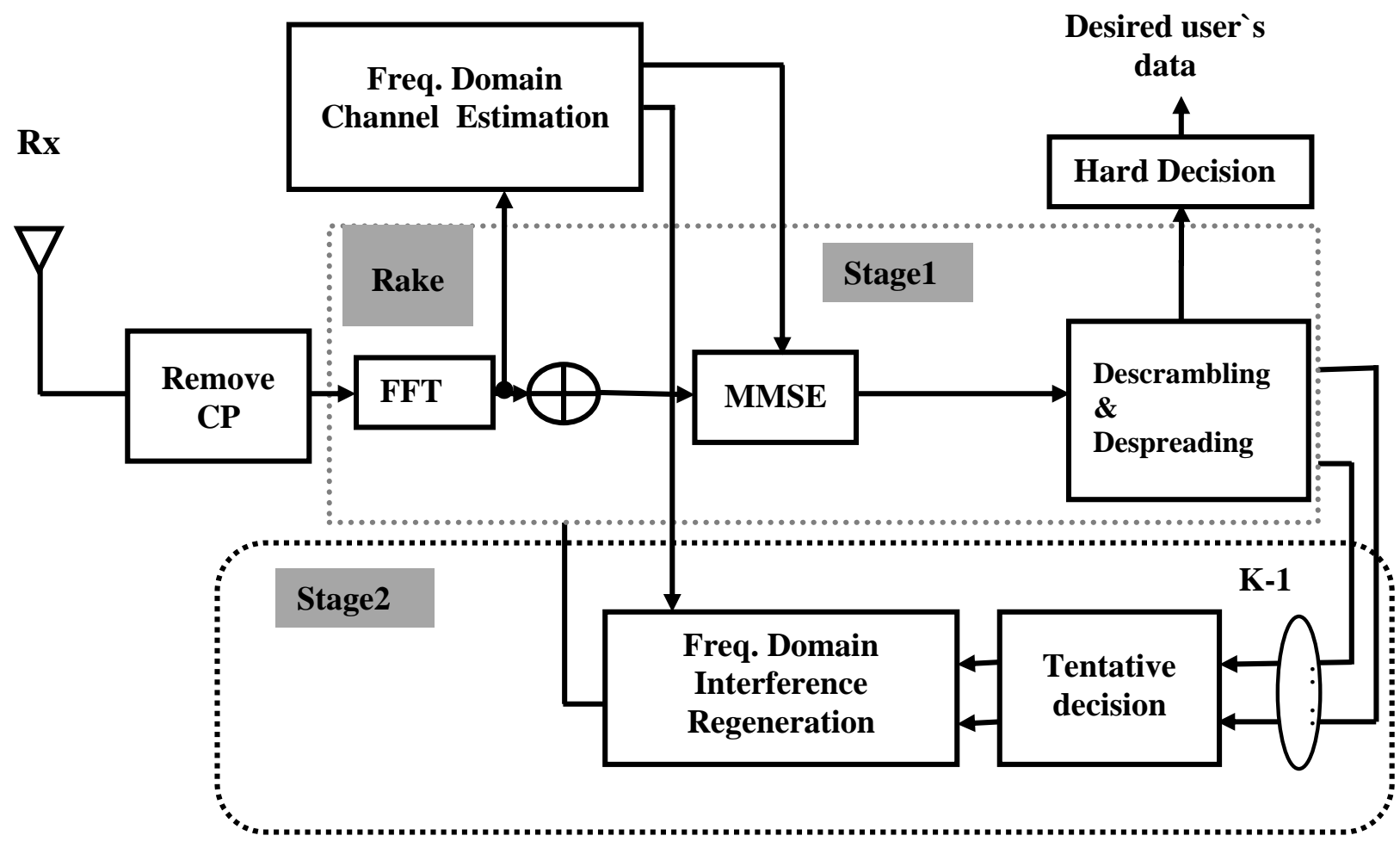

Fig. 3. Structure of the MMSE-PIC for downlink MC-CDMA systems.

$$
R_{M A I}=E \mathbf{F}^{-1}\left(\boldsymbol{C U} \hat{\mathbf{b}}_{\text {int }}\right)
$$

The MAI is then subtracted from $\mathbf{R}_{\mathrm{T}}$ to get a frequency domain interference free signal as follows:

$$
\boldsymbol{z}=\boldsymbol{R}_{T}-\boldsymbol{R}_{M A I}
$$

- A better estimate of the symbol of interest can be obtained after MMSE equalizer, descrambling, and despreading as follows:

$$
\hat{\mathbf{b}}_{\text {des }}=\boldsymbol{f}_{\text {dec }}\left\{C_{d e s}^{T} C^{H} W_{M M S E} Z\right\}
$$

The performance of the proposed MMSE-PIC scheme is the best as compared to the RAKEPIC receiver, especially when the number of users is high. However, its complexity is greater than that of the RAKE-PIC, receiver and the statistics of the additive noise and the transmitted data are required.

\section{Simulation Results}

Several simulation experiments are carried out in this section to test the performance of the proposed algorithms and compare them to each other. The simulation environment is based on the downlink synchronous MC-CDMA system in which each user transmits BPSK information symbols. The propagation channel is assumed to be a frequency selective fading channel with $L=3$ paths and a uniform delay profile. More details of the simulation parameters are given in Table 1. All users are assigned the same power.

Figure 4 illustrates the BER versus the threshold of the null zone decision function $\left(c_{n}\right)$ at different SNR values and different number of users for the MMSE-PIC algorithm. The 
observation from this figure is show that $c_{n}$ opt $=0.2$ is always the best choice regardless of the value of the SNR. Fig. 4 shows that $c_{n}$ opt is nonsensitive to SNR-changes and to systemload changes.

Table 1. Simulation parameters

\begin{tabular}{c||l|l}
\hline \hline \multirow{4}{*}{ Transmitter } & Modulation & BPSK \\
\cline { 2 - 3 } & Spreading Codes & Walsh-Hadamard codes with spreading factor 32 \\
\cline { 2 - 3 } & FFT points & $\mathrm{P}=256$ \\
\cline { 2 - 3 } Channel & Cyclic Prefix & $\mathrm{N}_{\mathrm{CP}}=32$ \\
\cline { 2 - 3 } & Fading & Frequency selective with L=3 \\
\hline \multirow{3}{*}{ Receiver } & Equalization & $\begin{array}{l}\text { MRC(RAKE), } \\
\text { and MMSE Equalizer }\end{array}$ \\
\cline { 2 - 3 } & Channel estimation & Ideal \\
\hline \hline
\end{tabular}

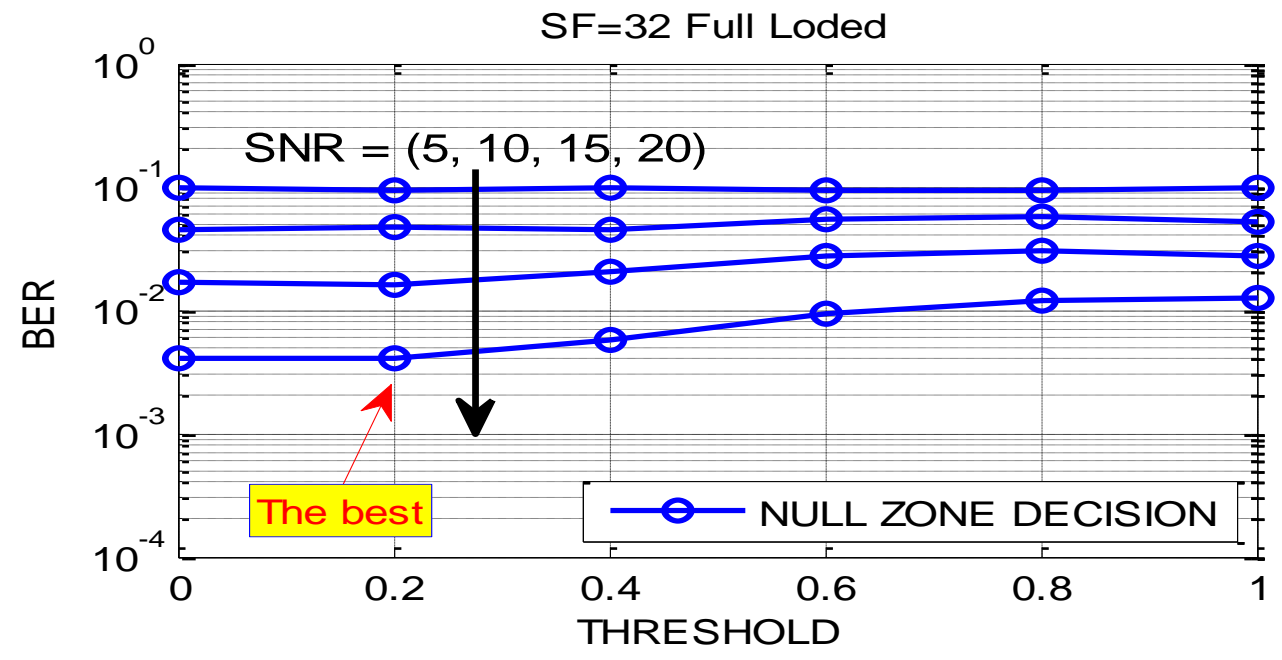

Fig. 4 BER vs Null Zone Decision Threshold at Different SNRs, $K=32$ (full load).

The effect of the tentative decision function on the performance of the RAKE-PIC algorithm for the loaded users $K=32$ (full load) is studied and shown in Fig. 5. It is found that the best performance of the RAKE-PIC algorithm can be obtained with the tanh, unit clipper and soft decision functions that have the same performance. However it has been shown that the NullZone decision gives the same performance as the three previously mentioned decisions at 25 $\mathrm{dB}$ of SNR. On the other hand, the effect of the tentative decision function on the performance of the MMSE-PIC algorithm for $K=32$ (full load) is studied and shown in Fig. 6. It is found that the best performance of the MMSE-PIC algorithm can be obtained with the hard and null zone decision functions. 


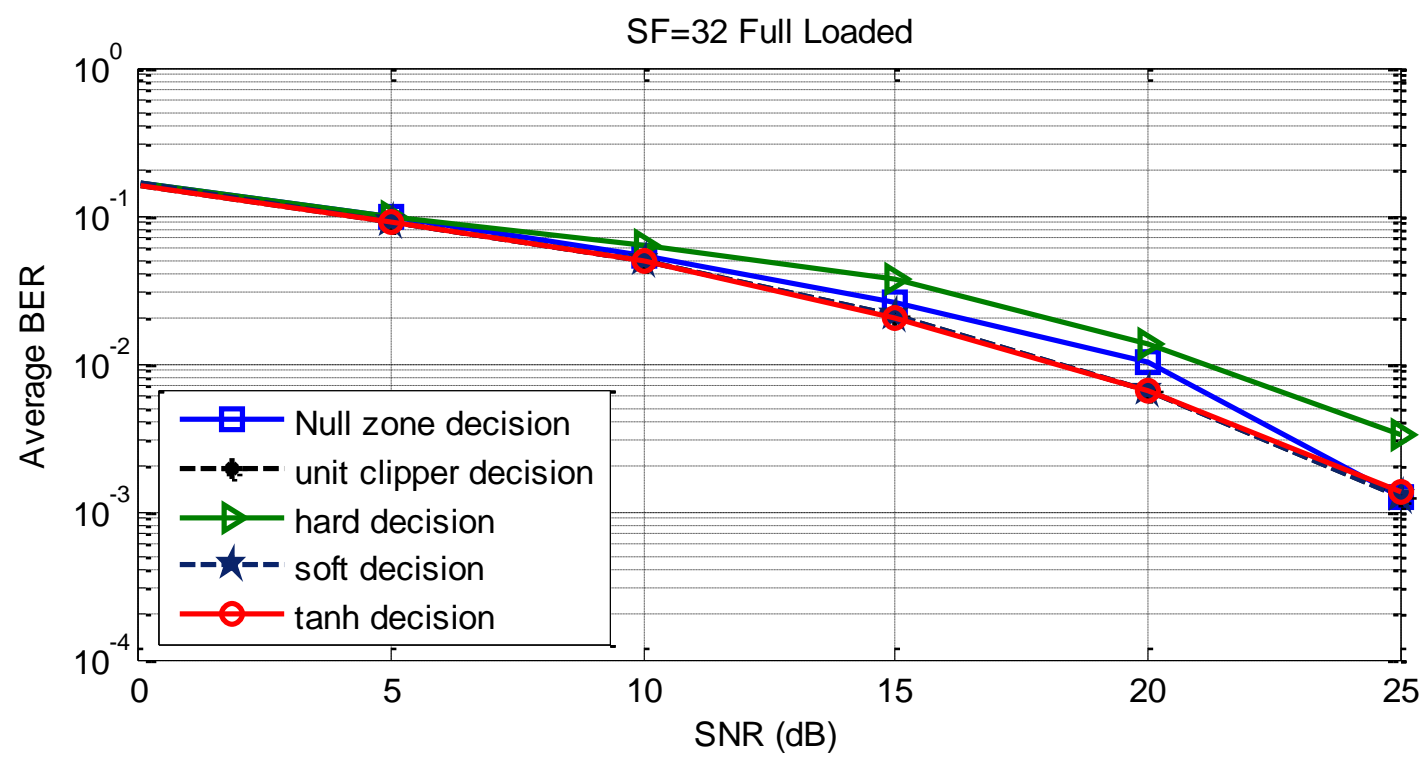

Fig. 5 Performance of different schemes with different decision functions of RAKE-PIC. for $K=32$ (full load)

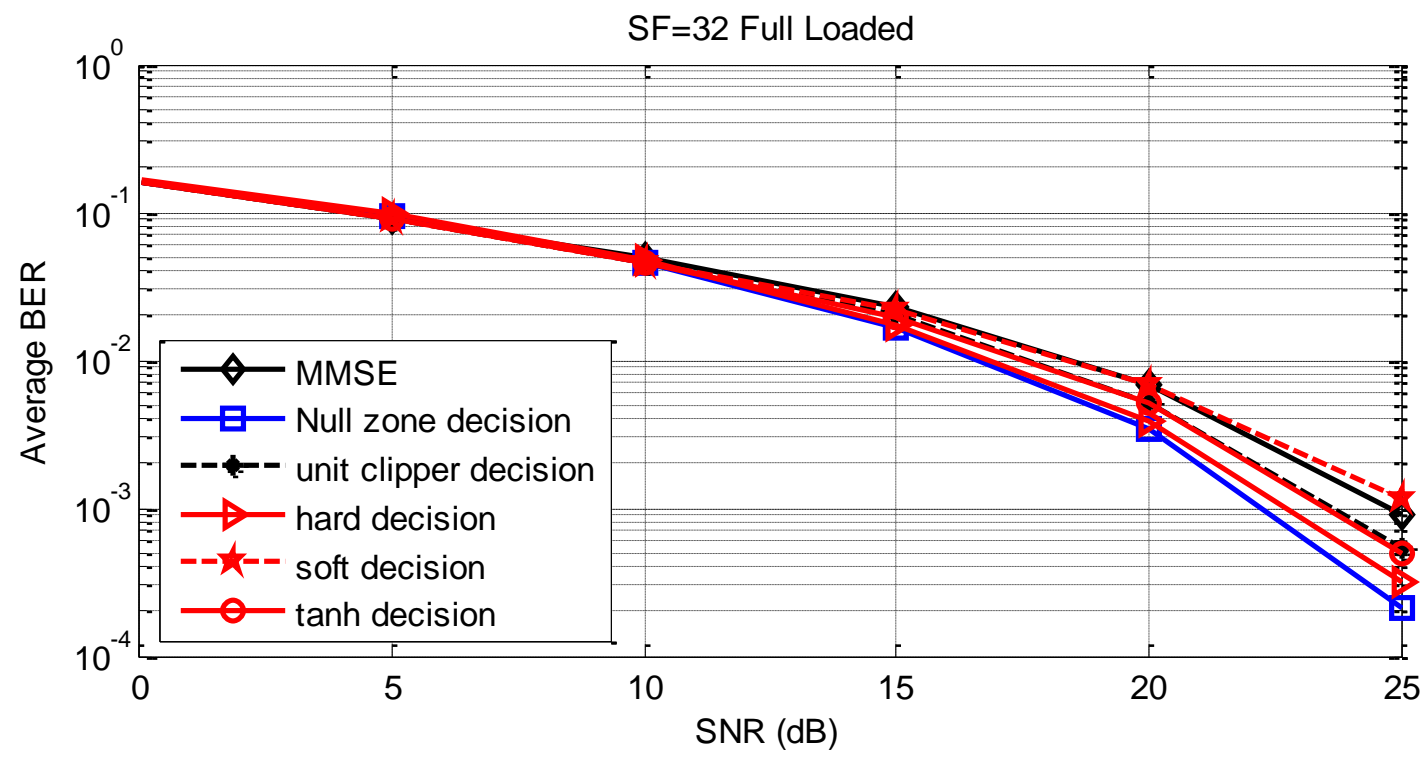

Fig. 6 Performance of different schemes with different decision functions of MMSE-PIC, for $\mathrm{K}=32$ (full load)

Figure 7 introduces comparisons between the three different proposed algorithms for $K=16$ and $K=32$, respectively. From the obtained results, it is clear that for low SNR values, the three proposed algorithms have the same performance. At high SNR values, the MMSE-PIC algorithm gives the best performance. From Fig. 7a, it can be observed that the performance of the RAKE-PIC and the MMSE algorithms is the same when the system load is low. This observation may be due to the propagation of errors.

When the number of users is small, the RAKE receiver provides reliable decisions and the resulting propagation of errors will be small and the MMSE-PIC gives good performance when the system load is high that shown in fig. $7 \mathrm{~b}$ at $\mathrm{K}=32$ (full load) the performance is the same as the performance in fig. $7 \mathrm{a}$ at $\mathrm{K}=16$ (half load) .

The effects of user loads on the performance of the MMSE-PIC algorithms are studied and presented in Fig. 8. 


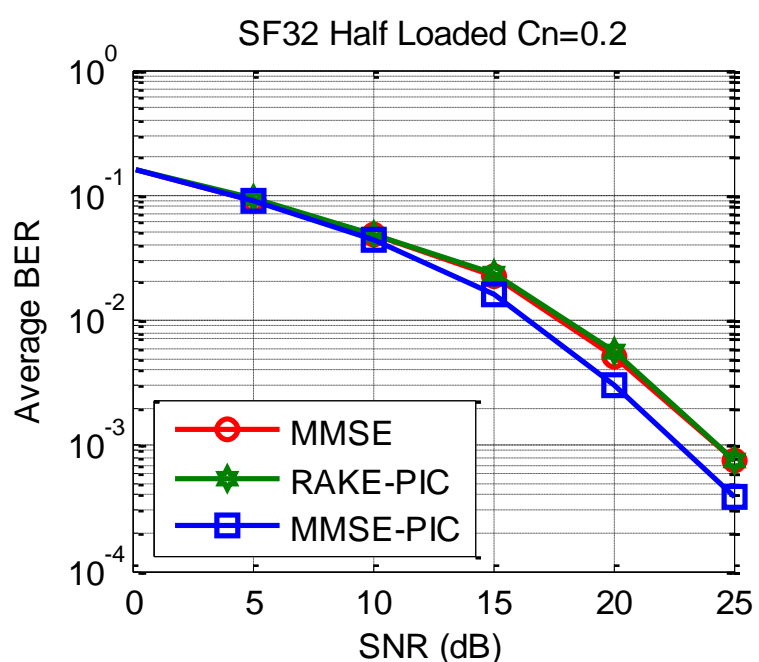

(a)

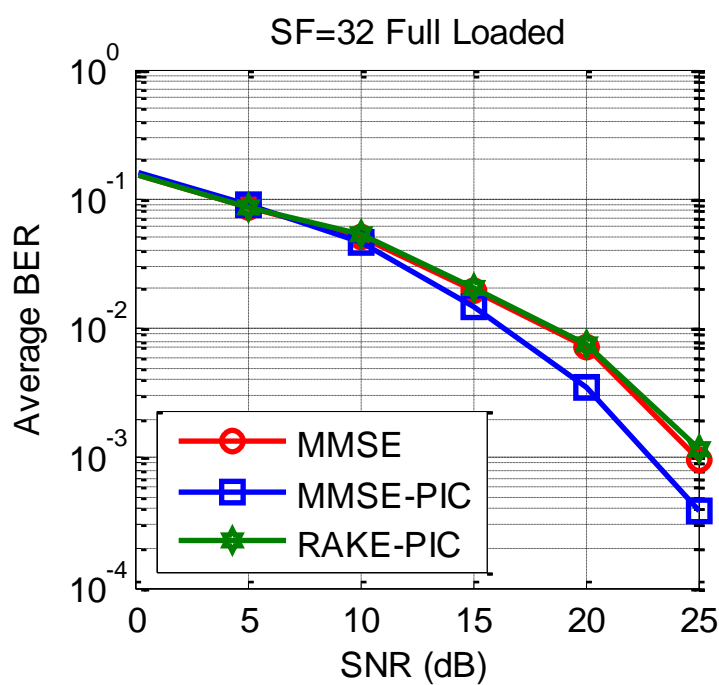

(b)

Fig. 7 BER vs SNR for different reception schemes.

(a) $K=16$ (half load). (b) $K=32$ (full load).

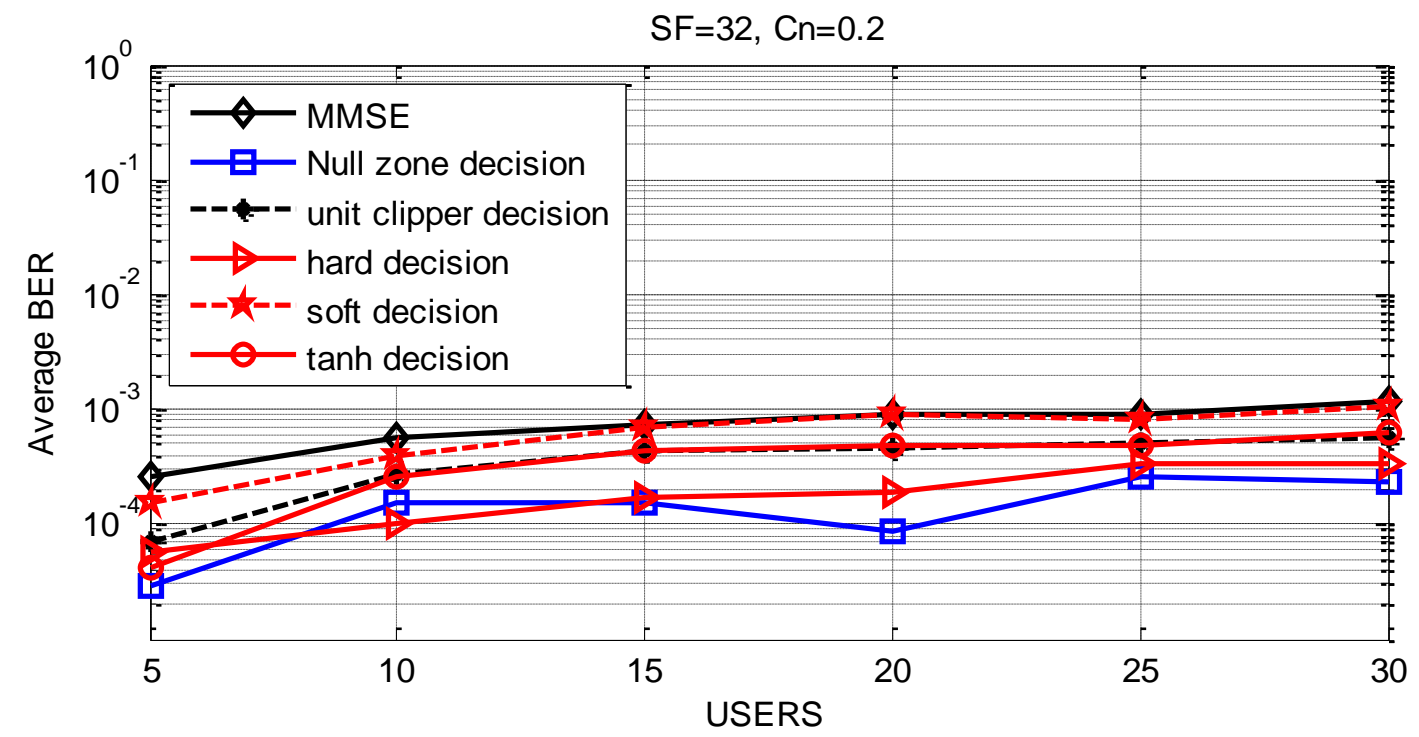

Fig. 8. BER vs the number of active users for the different tentative decisions for MMSE-PIC algorithm. SNR = $20 \mathrm{~dB}$.

The BER of all receivers degrades as the number of users increases. In this case, the performance of the suggested algorithms degrades a little bit by increasing the number of users, but it is still better than the other algorithms and the crossing of the tanh decision over the hard decision for users between (5-10), is occurred due the number of iteration of the simulation.

The effects of user loads on the performance of the suggested algorithms are studied and presented in Fig. 9. The BER of all receivers degrades as the number of users increases. This observation may be due to the MAI. The higher the number of users, the larger the MAI. Even after interference cancellation, some residual MAI still exists. Therefore, the performance loss may be attributed to the residual MAI. From these results the performance of the MMSE-PIC 
algorithm degrades a little bit by increasing the number of users, but it is still better than the RAKE-PIC and MMSE algorithms.

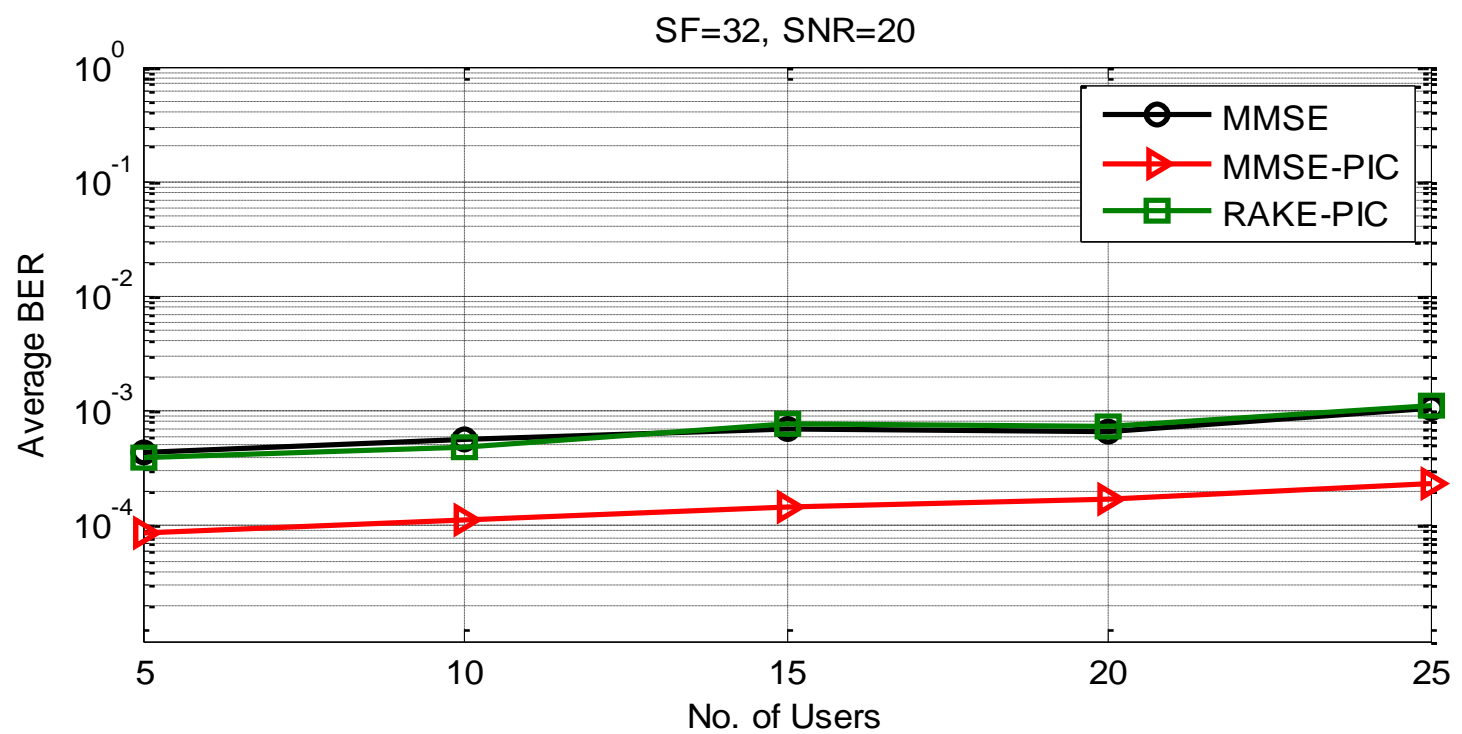

Fig. 9. BER vs the number of active users for the different reception schemes. $\mathrm{SNR}=\mathbf{2 0} \mathrm{dB}$.

Figure 10 depicts the BER versus the number of canceled users, at a fixed SNR per user of 20 $\mathrm{dB}$. This figure shows that the performance of the suggested algorithms improves as the number of canceled users increases.

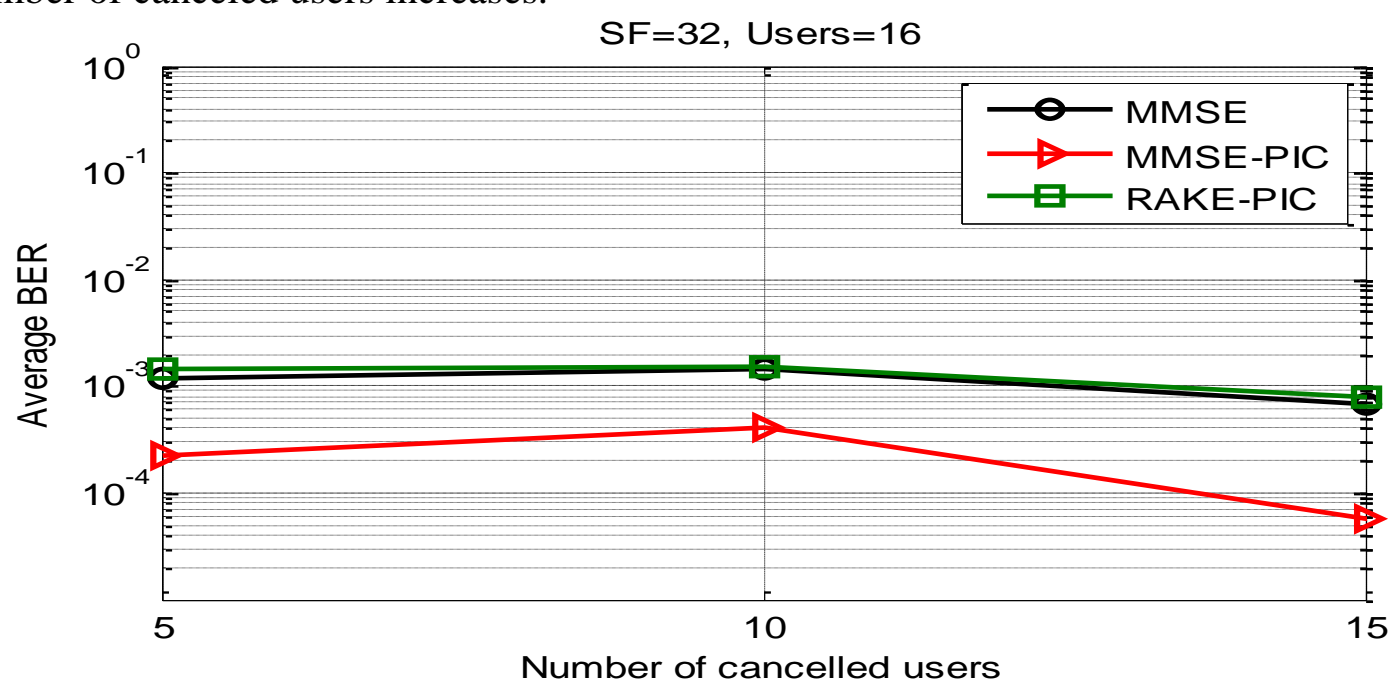

Fig. 10 BER vs the number of canceled users for the different reception schemes, $\mathrm{SNR}=20 \mathrm{~dB}$.

\section{Conclusions}

In this paper, two interference cancellation algorithms have been suggested and studied to suppress the interference for downlink MC-CDMA systems. The comparison studies show that the suggested algorithms achieve performance improvement compared to the traditional MMSE equalizer and the RAKE receiver algorithms. The effects of the tentative decision function used, the number of users, and the numbers of canceled users on the performance of these algorithms are studied. It is found that, the MMSE-PIC with hard decision and null zone 
decision provide better performance than other algorithms even at heavy loads. This indicates that a reliable communication is possible with the proposed algorithms.

\section{References}

[1] Wei Yang, Jun-Ying Liu, Shi-Xin Cheng, Effect of carrier-frequency offset on the performance of group orthogonal multicarrier CDMA systems, Signal Processing 86 (2006), pp. 3934-3940.

[2] Jun-Bo Wang, Ming Chen, Jiangzhou Wang, Adaptive channel and power allocation of downlink multi-user MC-CDMA systems, Computers and Electrical Engineering, 35 (2009), pp. 622-633.

[3] Carlos J. Escudero, Daniel I. Iglesia, Luis Castedo., Blind subspace methods for code and channel estimation in Multicarrier CDMA., Signal Processing 83 (2003), pp. 339 $-357$.

[4] Yun Rui, Honglin Hu, Huiyue Yi, Hsiao-Hwa Chen, Yueh-Min Huang, Frequency domain discrete fourier transform spread generalized multi-carrier system and its performance analysis., Computer Communications 32 (2009), pp. 456-464.

[5] F. Adachi, D. Garge, S. Takaoka, K. Takeda, Broadband CDMA techniques, IEEE Wireless Commun. 12 (2) (2005), pp. 8-18.

[6] H. J. Taha and M. F. M. Salleh, Multicarrier transmission techniques for wireless communication systems: a survey, WSEAS Trans. Communications, issue 5, Vol. 8, 2009, pp. 457- 472.

[7] Y. J. Chiu, Adaptive MMSE Rake-Equalizer receiver design with channel estimation for DS-UWB system, WSEAS Trans. Communications, issue 1, Vol. 8, 2009, pp.196205.

[8] S. Hara, and R. Prasad, Design and performance of multicarrier CDMA system in frequency-selective Rayleigh fading channels, IEEE Trans. Vehicular Technology., Vol. 48, No. 5, 1999, pp.1548-1595.

[9] J. I. Chen, and Y. Su, Designing and evaluation of an MC-DS-CDMA system with dual dimension Rake receiver, Proceeding of the 11th WSEAS International Conference on Communications, Agios Nicolaos, Crete island, Greece, July 26-28, 2007, pp.178-183.

[10] F. S. Al-kamali, M. I. Dessouky, B. M. Sallam, and F. E. El-Samie, "Performance of Cyclic Prefix CDMA Systems with Frequency Domain Interference Cancellation," submitted for publication in the digital signal Processing Journal, Elsevier Inc.2009, pp. 2-13.

[11] K. Takeda, F. Adachi, Inter-chip interference cancellation for DS-CDMA with frequency domain equalization, in: IEEE VTC, vol. 4, 26-29 Sept. 2004, pp. 23162320.

[12] K. Takeda, K. Ishihara, F. Adachi, Downlink DS-CDMA transmission with joint MMSE equalization and ICI cancellation, in: IEEE VTC, vol. 4, Spring 2006, pp. $1762-1766$.

[13] K. Takeda, F. Adachi, Frequency-domain interchip interference cancellation for DSCDMA downlink transmission, IEEE Trans. Veh. Technol. 56 (3) (2007), pp. 12861294.

[14] Y.C. Liang, Block-iterative GDFE (BI-GDFE) for CP-CDMA and MC-CDMA, in: IEEE VTC, vol. 5 (6), 30 May-1 June 2005, pp. 3033-3037.

[15] Y. Liang, S. Sun, C. Ho, Block-iterative generalized decision feedback equalizers (BI-GDFE) for large MIMO systems: Algorithm design and asymptotic performance analysis, IEEE Trans. Signal Process. 54 (6) (2006), pp. 2035-2048. 
[16] S. Tomasin, N. Benvenuto, Frequency domain interference cancellation and nonlinear equalization for CDMA systems, IEEE Trans. Wireless Commun.4 (5) (2005), pp. 2329-2339.

[17] I.A. Krikidis, Parallel interference cancellation for DS-CDMA downlink with low spreading factors, in: IEEE Workshop on Signal Processing Systems. Design and Implementation, 2-4 Nov. 2005, pp. 699-704.

[18] F.S. Al-kamali, M.I. Dessouky, B.M. Sallam, F.E. El-Samie, Parallel interference cancellation and linear equalization for multirate downlink CDMA systems, in: ICCTA 2007, Alexandria, Egypt, 1-3 Sept. 2007, pp. 180-186.

[19] A.L.C. Hui, K.B. Letaief, Multiuser asynchronous DS/CDMA detectors in multipath fading links, IEEE Trans. Commun. 46 (1998), pp. 384-391.

[20] L.B. Nelson, H.V. Poor, Iterative multiuser receivers for CDMA channels: An EMbased approach, IEEE Trans. Commun. 44 (1996) 1700-1710.]

[21] S. Werner, J. Lilleberg, Downlink channel decorrelation in CDMA systems with long codes, in: IEEE 49th VTC, vol. 2, 16-20 May 1999, pp. 1614-1617. 